Supplement 1. C1s Curve fits of oligoglyme and crown ether plasma deposited films (PDFs)

\title{
Monoglyme PDF
}

\begin{tabular}{|c|c|}
\hline EV max & \% area \\
\hline 285.01 & 33.03 \\
\hline 286.53 & 56.02 \\
\hline 287.87 & 8.8 \\
\hline 289.37 & 2.15 \\
\hline
\end{tabular}






\section{Diglyme PDF}

\begin{tabular}{|c|c|}
\hline EV max & \% area \\
\hline 284.99 & 61.03 \\
\hline 286.60 & 30.46 \\
\hline 287.92 & 6.64 \\
\hline 289.31 & 1.86 \\
\hline
\end{tabular}

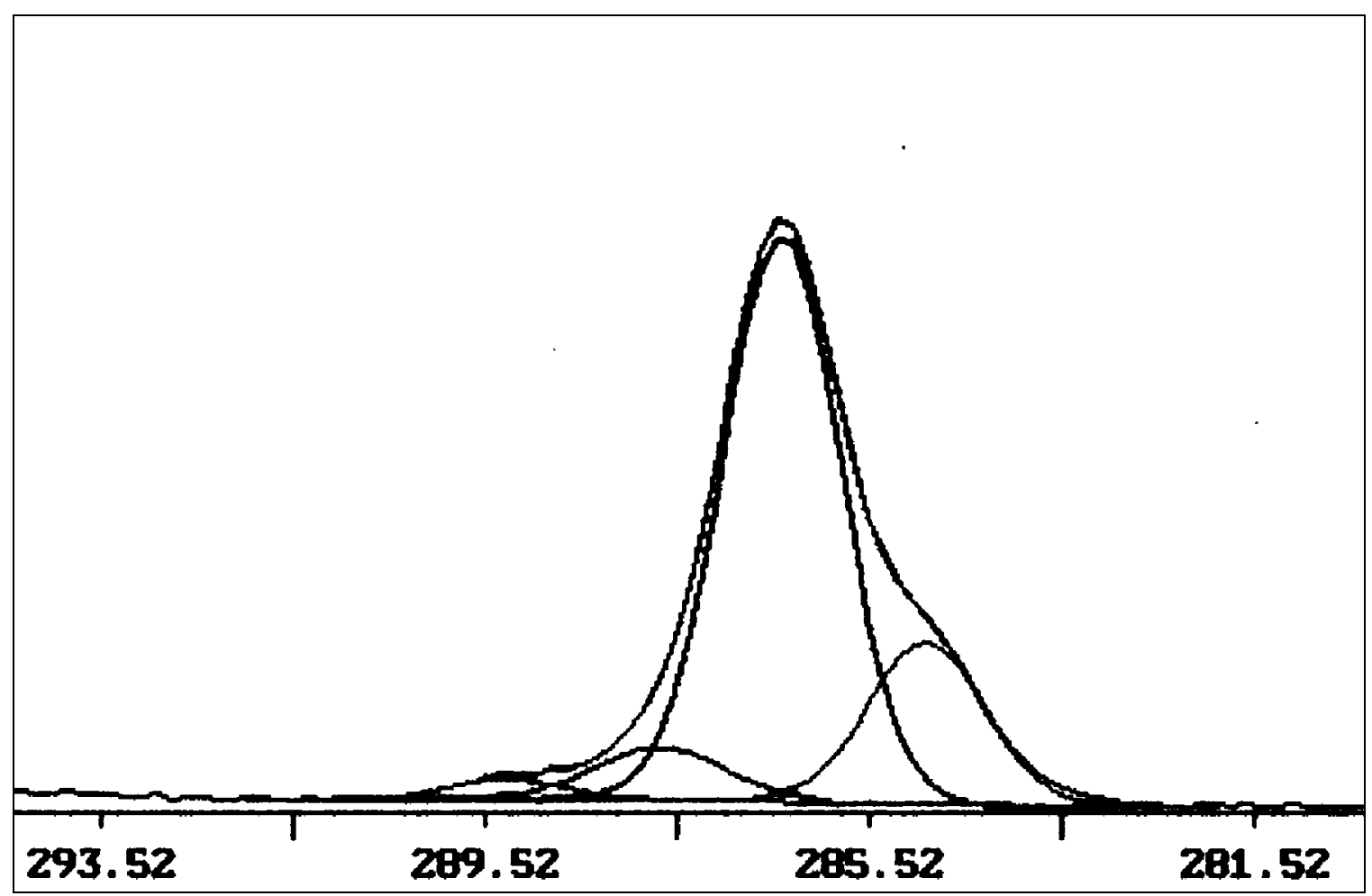


Triglyme PDF

\begin{tabular}{|c|c|}
\hline EV max & \% area \\
\hline 286.58 & 68.27 \\
\hline 285.05 & 23.58 \\
\hline 288.04 & 6.27 \\
\hline 289.49 & 1.89 \\
\hline
\end{tabular}

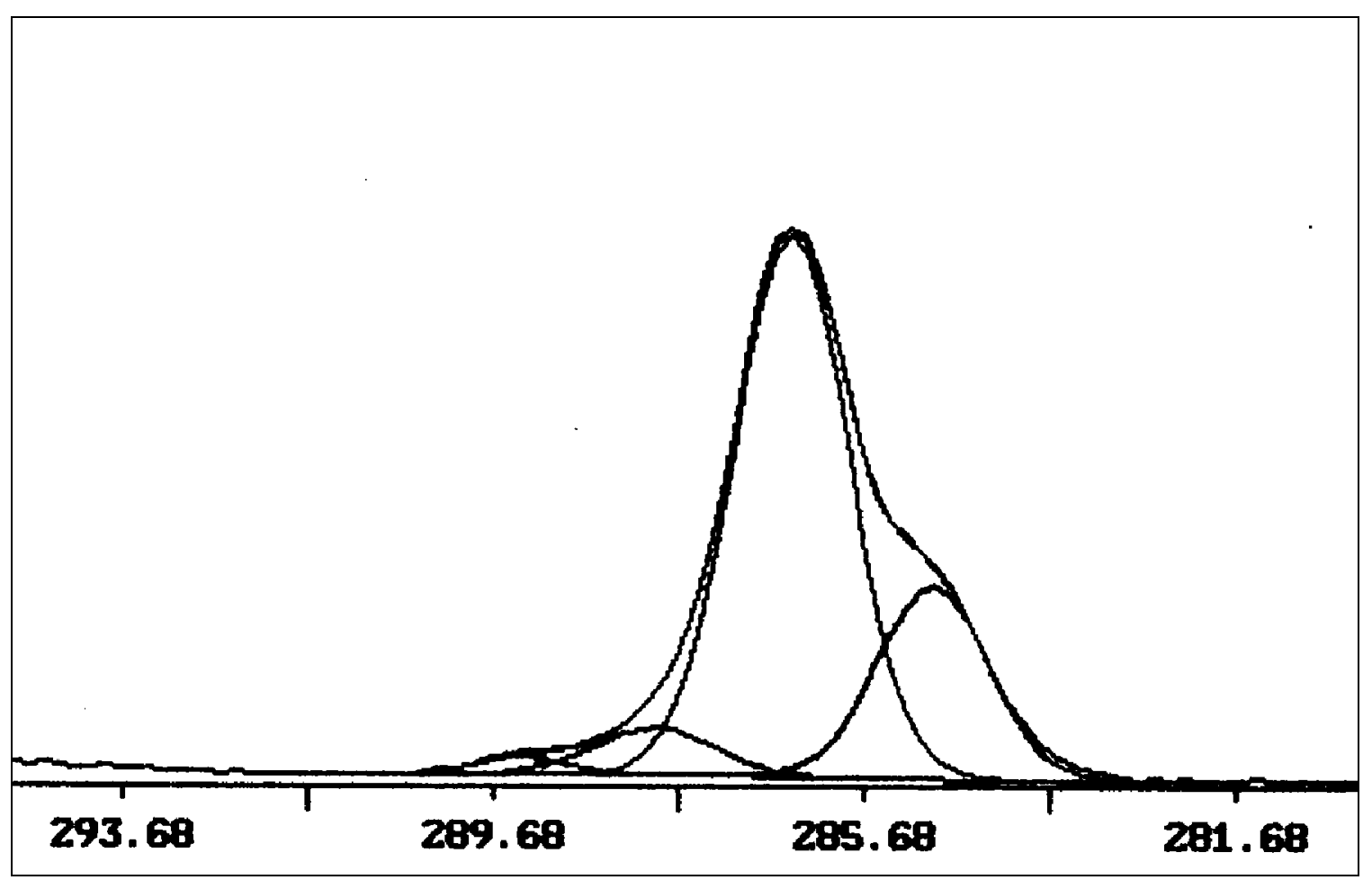


Tetraglyme, 5w PDF

\begin{tabular}{|c|c|}
\hline EV max & \% area \\
\hline 286.65 & 85.00 \\
\hline 285.15 & 10.45 \\
\hline 288.08 & 3.59 \\
\hline 289.52 & 0.96 \\
\hline
\end{tabular}

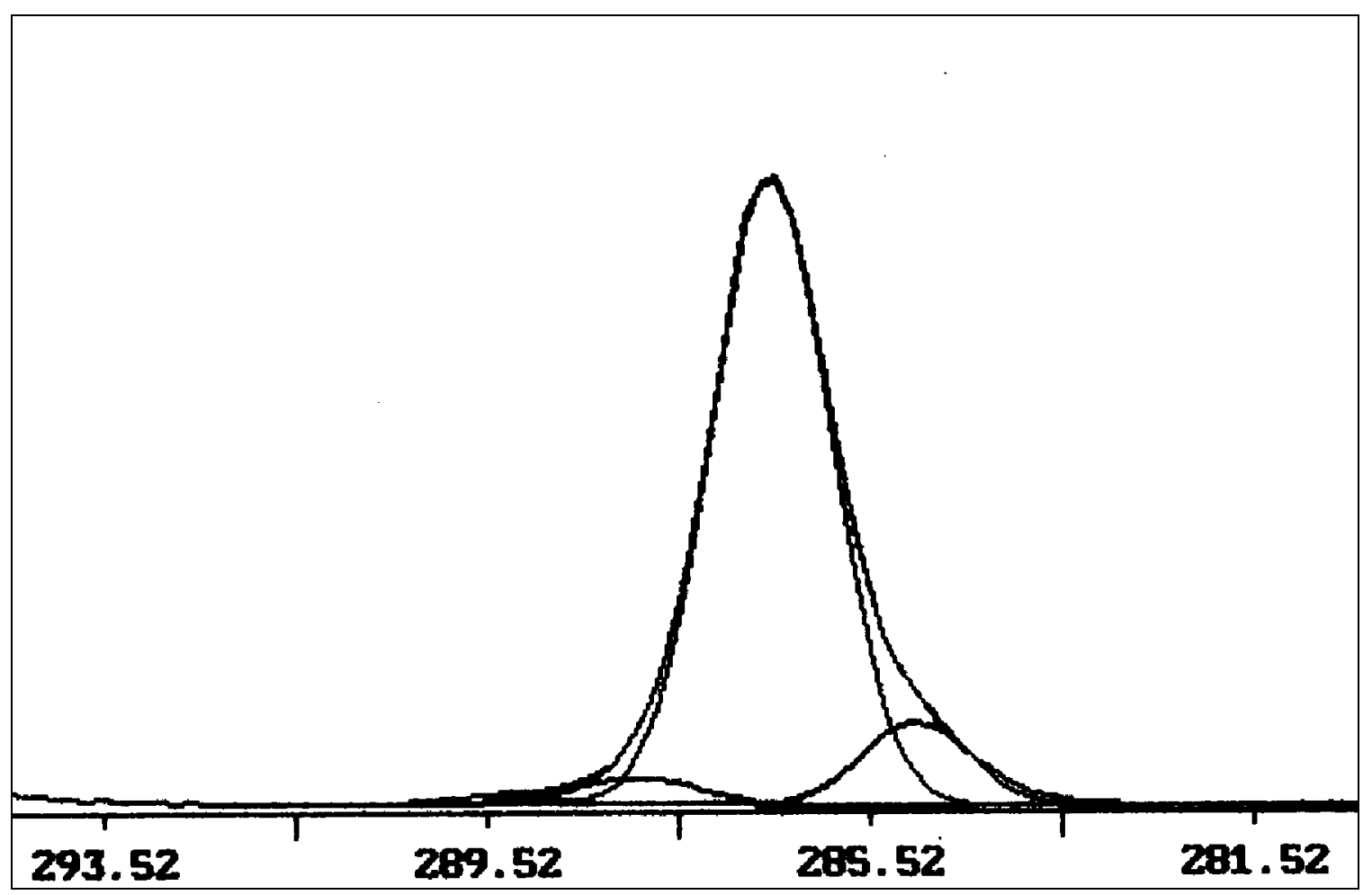


Tetraglyme, 20w PDF

\begin{tabular}{|c|c|}
\hline EV max & \% area \\
\hline 286.62 & 84.11 \\
\hline 285.13 & 10.78 \\
\hline 287.96 & 3.68 \\
\hline 289.38 & 1.43 \\
\hline
\end{tabular}






\section{Dioxane PDF}

\begin{tabular}{|c|c|}
\hline EV max & \% area \\
\hline 284.99 & 61.03 \\
\hline 286.60 & 30.46 \\
\hline 287.92 & 6.64 \\
\hline 289.31 & 1.86 \\
\hline
\end{tabular}

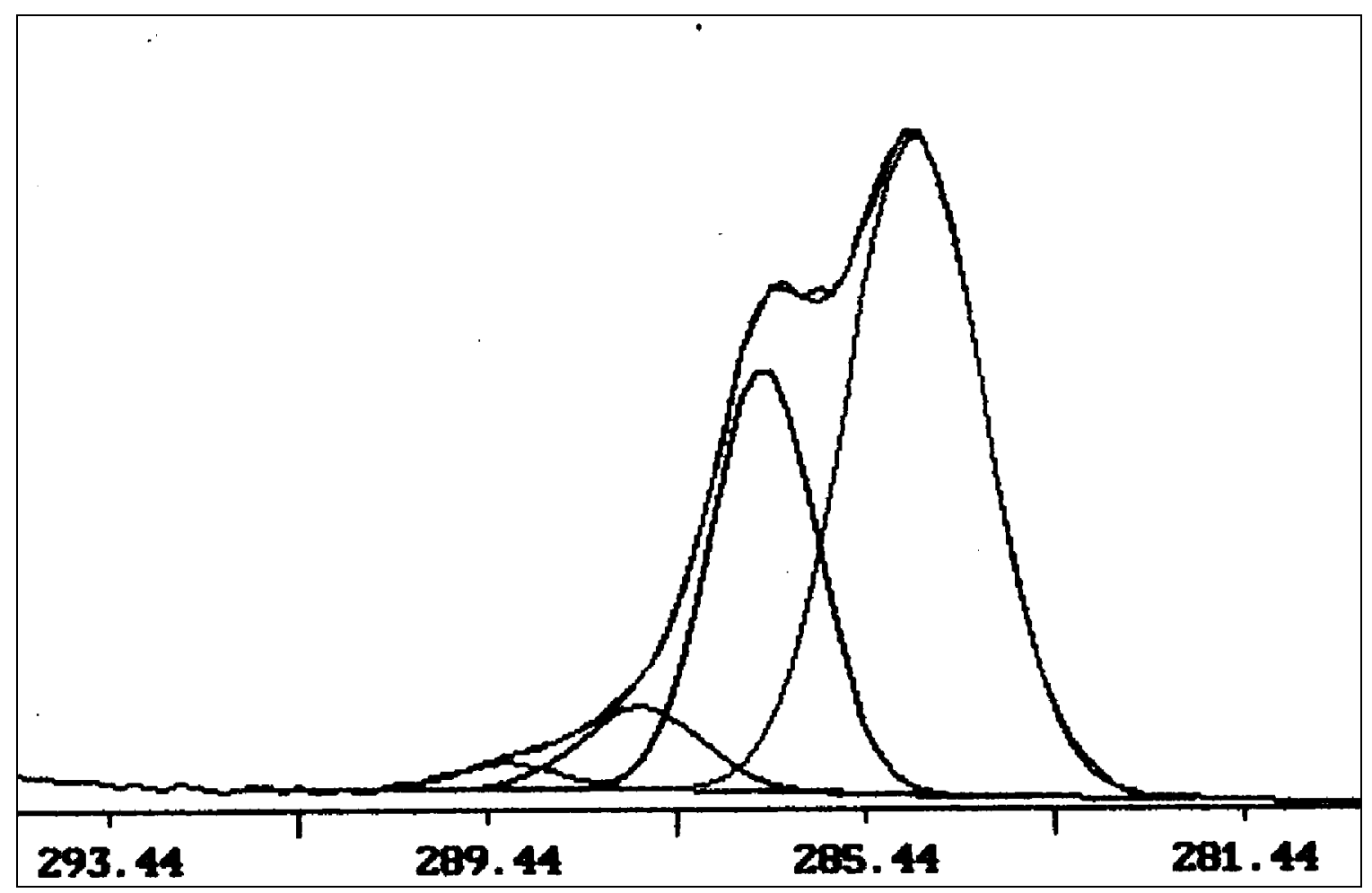




\section{2-Crown-4 ether PDF}

\begin{tabular}{|c|c|}
\hline EV max & \% area \\
\hline 286.53 & 79.05 \\
\hline 285.05 & 14.96 \\
\hline 288.11 & 3.44 \\
\hline 289.36 & 2.82 \\
\hline
\end{tabular}

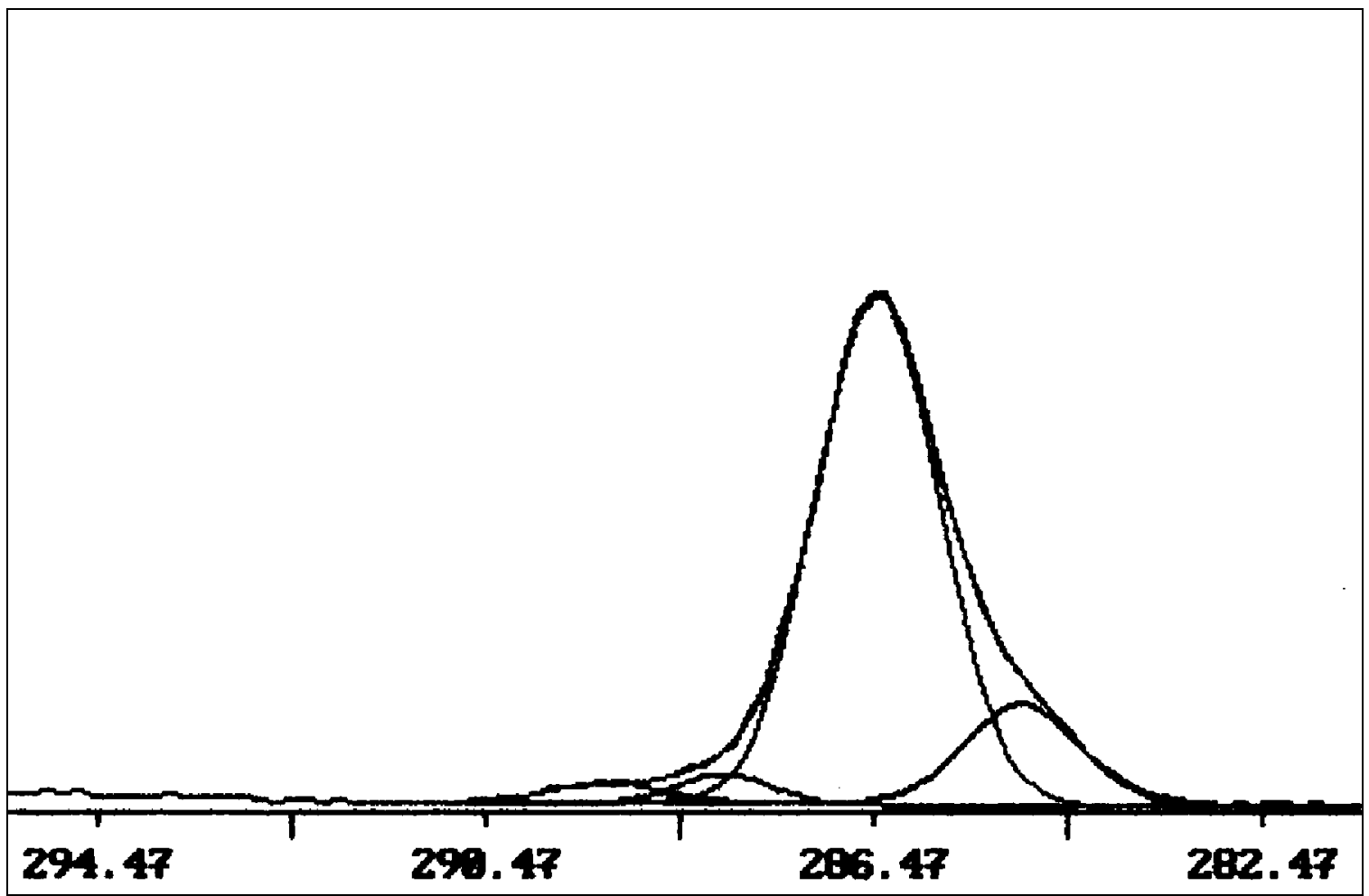




\section{5-Crown-5, 5w PDF}

\begin{tabular}{|c|c|}
\hline EV max & \% area \\
\hline 286.57 & 78.59 \\
\hline 285.10 & 14.34 \\
\hline 288.07 & 5.28 \\
\hline 289.46 & 1.79 \\
\hline
\end{tabular}

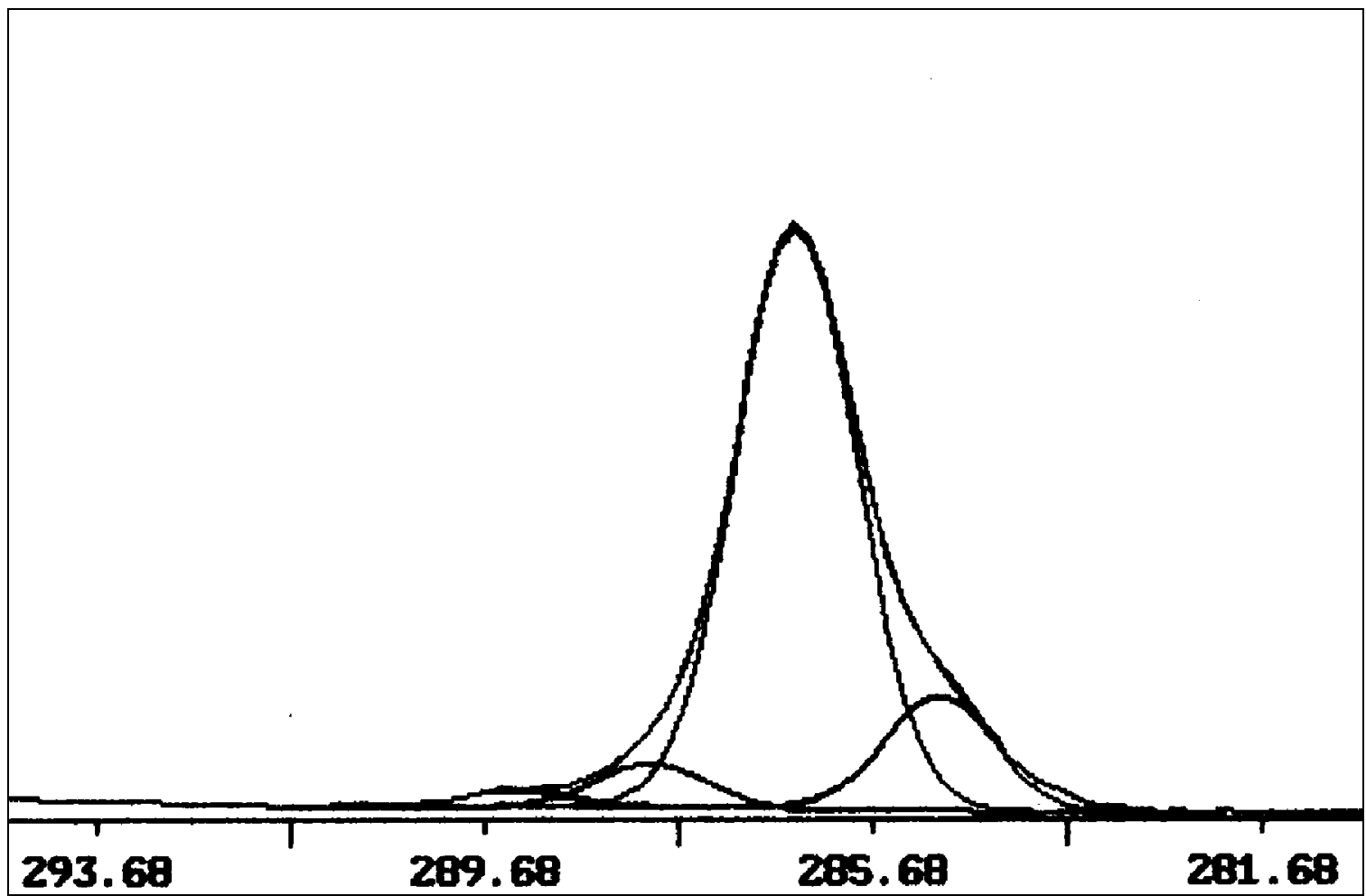


15-Crown-5, 20w PDF

\begin{tabular}{|c|c|}
\hline EV max & \% area \\
\hline 286.51 & 84.45 \\
\hline 285.05 & 9.70 \\
\hline 287.88 & 5.06 \\
\hline 289.39 & 0.79 \\
\hline
\end{tabular}

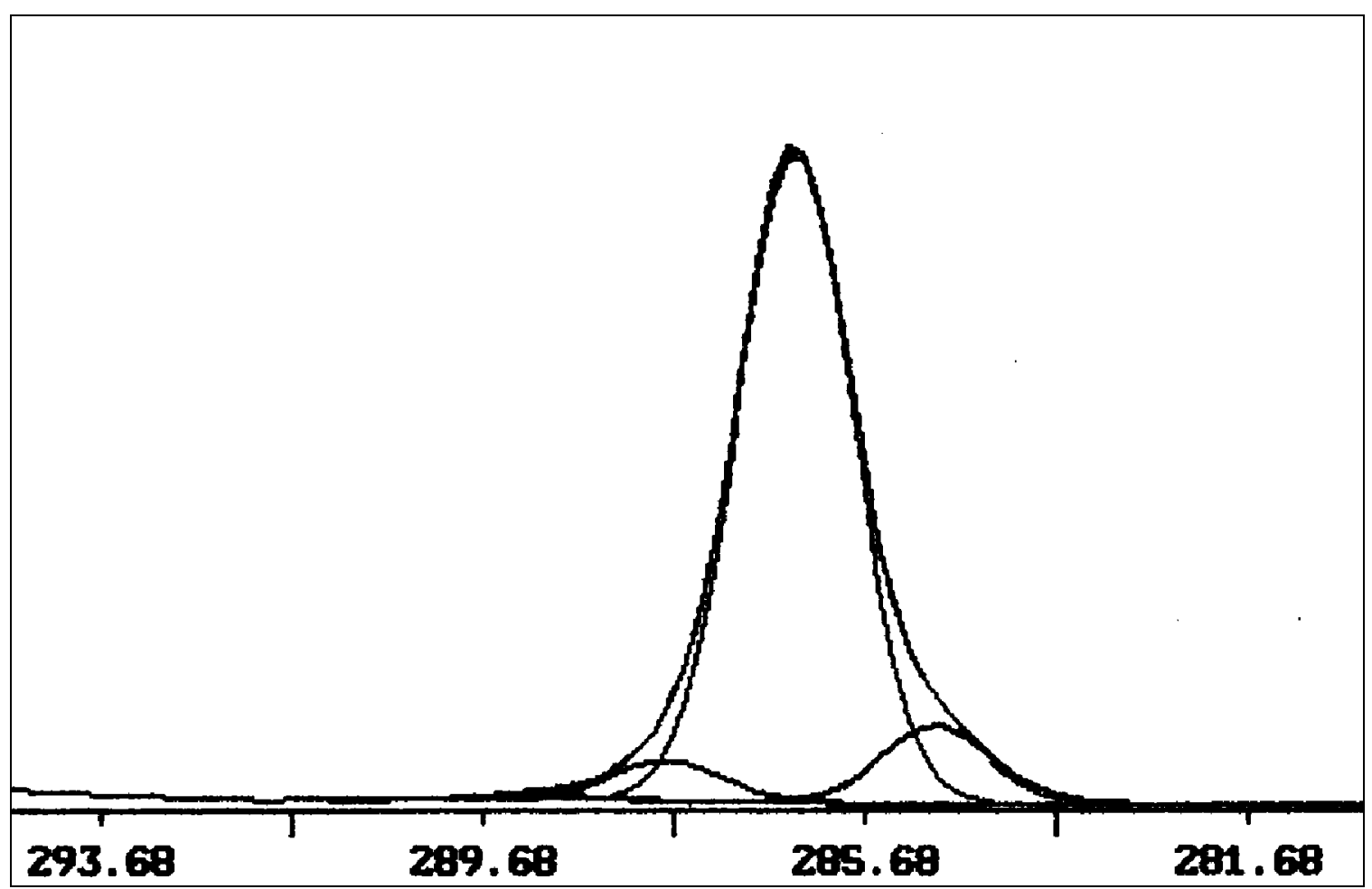


